\title{
Effect of Streptozotocin-Induced Hyperglycemia on the Progression of Hepatocarcinogenesis in Rats
}

\author{
Chowdhury Mobaswar Hossain, Bhabani Sankar Satapathy, \\ Laboni Mondal, Samrat Chakraborty and Biswajit Mukherjee \\ Department of Pharmaceutical Technology, \\ Faculty of Engineering and Technology, Jadavpur University, Kolkata, India
}

Received 2013-10-13, Revised 2013-11-01; Accepted 2013-11-04

\begin{abstract}
Diabetes mellitus and hepatocellular carcinoma both have detrimental impact on health worldwide. Type II diabetes and liver cancer share many causative factors, but biological correlation between the two diseases still remains elusive. The study was aimed to evaluate the effect of induction of diabetes during the development of hepatocarcinogenesis in rats. Rats were divided into four groups namely, normal control, diabetic control, carcinogen control and carcinogen treated rats treated with streptozotocin (to make them diabetic). Hepatocarcinogenesis was initiated in rats by diethylnitrosamine $\left(200 \mathrm{mg} \mathrm{kg}^{-1}\right.$ body weight, single i.p. injection on day 0 only). Then 2 -acetylaminoflourene $(0.5 \% \mathrm{w} / \mathrm{w})$ was given daily in diet for 18 weeks to promote the carcinogenesis. On the 16th week, streptozotocin $\left(65 \mathrm{mg} \mathrm{kg}^{-1}\right.$ body weight, single i.p. injection) was administered to initiate diabetes in rats. On the 20th week, animals were sacrificed and various biochemical changes and histopathological alterations in liver were investigated. Carcinogen treated rats made diabetic had significantly lower cytochrome P-450 content as compared to diabetic control rats and had slightly elevated cytochrome P-450 level as compared to that of carcinogen control rats. Marked enhancements of UDP-glucuronosyl transferase, glutathione S-transferase activities and lipid peroxidation levels were observed in carcinogen treated rats made diabetic as compared to those activities and levels in diabetic control and carcinogen control rats. Histopathological investigation of hepatic tissue has favoured the rapid progress of development of hepatocellular carcinoma in carcinogen treated rats made diabetic. In conclusion, induction of diabetes during the development of hepatocellular carcinogenesis inevitably promotes the progression of the later disease.
\end{abstract}

Keywords: Diabetes, Hepatocellular Carcinogenesis, Preneoplastic/Neoplastic Lesion

\section{INTRODUCTION}

Hepatocellular Carcinoma (HCC) is the fifth most common cancer and the third most common cause of cancer-related death in the world (Thomas et al., 2010). HCC occurs due to viruses (hepatitis B virus and hepatitis $\mathrm{C}$ virus), chemical carcinogens (such as aflatoxins, nitrosamine) and other environmental and host factors causing chronic hepatocellular injury (Liu and $\mathrm{Wu}$, 2010). The process of hepatocarcinogenesis is complex, resulting from alterations in the normal patterns of cellular growth. The development of HCC is believed to occur due to a distorted expression of thousands of genes. It is preceded by the early appearance of morphologically and genetically altered "resistant cellular phenotypes" termed as hepatic focal lesions or preneoplastic lesions (Das et al., 2010). Diabetes mellitus (DM) is one of the most common endocrine diseases in the world characterized by the state of hyperglycaemia and affects multiple organs. It is a systemic disease caused by defect in the insulin secretion, insulin action or even both. The worldwide

Corresponding Author: Biswajit Mukherjee, Department of Pharmaceutical Technology, Faculty of Engineering and Technology, Jadavpur University, Kolkata, India Tel/Fax: +913324146677 
prevalence of diabetes in all age groups has been estimated to rise approximately $4.4 \%$ of the population or 552 million people by 2030 (Whiting et al., 2011). Diabetes and HCC are common diseases with tremendous impact on health. Worldwide, cancer and diabetes are the second and twelfth leading causes of death, respectively (Giovannucci et al., 2010). Epidemiologic evidence suggests that people with diabetes are at significantly higher risk for many forms of cancer (Giovannucci et al., 2010). The relative risks imparted by diabetes are greatest (about two folds or higher) for liver cancer (Simon and Balkau, 2010). Type II diabetes and liver cancer share many risk factors, but potential biological link between the two diseases is incompletely understood (Giovannucci et al., 2010). There is no convincing study available so far to establish the correlation between liver cancer and type II diabetes in a defined model. In this experiment, efforts have been made to study whether DM influences or more precisely promotes liver cancer progression and development in a defined animal model, to provide a more vivid understanding of possible link between these two diseases. This information may be relevant for the prevention and optimal patient management in case of the two dreadful diseases.

\section{MATERIALS AND METHODS}

\subsection{Chemicals and Reagents}

Diethylnitrosamine (DENA), 2-Acetylaminofluorene (2-AAF) and Streptozotocin (STZ) were purchased from Sigma Aldrich, Bangalore, India. Modified Lowry protein assay kit was obtained from Thermo scientific, Rockford, USA. All other chemicals, reagents and solvents used in this study were of analytical grade.

\subsection{Development of Rat Cancer Model}

Male Sprague-Dawley rats were purchased from Indian Institute of Chemical Biology, Kolkata, West Bengal. Initially the body weights of animals were approximately 110-130 g. All animals were kept in polypropylene cages and housed in the university animal house at $25 \pm 1{ }^{\circ} \mathrm{C}$ and $55 \%$ relative humidity environment with normal day and night photoperiod. Animals were fed standard rat diet and drinking water ad libitum. They were acclimatized to the animal house environment for 2 weeks. The animal experiments were designed and conducted upon approval of the Animal Ethics Committee (AEC), Jadavpur University,
Kolkata, India and strictly in accordance with the guidelines of AEC.

Rats were divided into four groups each containing six rats-Group I (normal control rats), Group II (diabetic control rats), Group III (carcinogen control rats) and Group IV (carcinogen treated rats treated with STZ).

At the start of the experiment (i.e., day 0), hepatocarcinogenesis was initiated in groups III and group IV animals by a single intraperitoneal (i.p.) injection of DENA at a dose of $200 \mathrm{mg} \mathrm{kg}^{-1}$ body weight (Bishayee et al., 2011). The animals were then treated with $0.5 \% \mathrm{w} / \mathrm{w}$ of 2 -AAF daily for 18 weeks (i.e., from 3 rd week to 20 th week) in the diet to promote carcinogenesis (Das et al., 2010). 2-AAF was mixed with a small amount of food and given every morning (Das et al., 2010). After $6 \mathrm{~h}$, the animals were given the basal diet (Das et al., 2010). The rats had free access to water. In group II and group IV rats (kept fasted overnight), induction of DM was started on 16 th week by a single i.p. injection (at a dose of 65 $\mathrm{mg} \mathrm{kg} \mathrm{k}^{-1}$ body weight) of freshly prepared solution of STZ in $0.1 \mathrm{M}$ citrate buffer (pH 4.5) (Liu et al., 2012). Daily food and water consumptions were observed (data not shown) and body weights were taken on a weekly basis. At the 20th week, when the rats were hyperglycemic with a mean blood glucose level 390

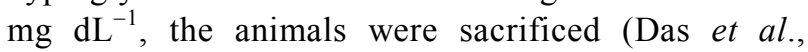
2010) and further experiments were conducted. All rats remained unfed for $12 \mathrm{~h}$ before being killed. The livers were removed, sliced (5-10 mm thick) and were snap-frozen in liquid nitrogen. The tissues were stored at $-80^{\circ} \mathrm{C}$ in ultra low temperature freezer (So-Low, Cincinnati, Ohio, USA) until further use.

\subsection{Histopathological Studies}

For histopathological studies, small pieces of liver (stored at $-80^{\circ} \mathrm{C}$ ) of the different experimental rats were taken out, thawed, fixed in $4 \%$ paraformaldehyde in phosphate buffer $(\mathrm{pH}$ 7.4) for 1 $\mathrm{h}$, subsequently processed and embedded in paraffin wax. Thin sections $(5 \mu)$ were cut and mounted on glass slides, processed for staining using haematoxylin-eosin ( $\mathrm{H}$ and $\mathrm{E}$ ) dye. The tissues were examined under Carl Zeiss light microscope (Axiostar plus, Jena, Germany).

\subsection{Determination of Mean Body Weight}

Body weights of rats from all the groups were taken on a weekly basis and the mean body weights for each group of rats were determined. 


\subsection{Biochemical Enzymes Assays}

\subsubsection{Preparation of Cytosolic and Microsomal Fraction}

Frozen liver tissue samples (stored at $-80^{\circ} \mathrm{C}$ ) of different experimental groups of rats were thawed, blotted quickly and weighed. The hepatic tissues were separately homogenized with ice-cold $0.1 \mathrm{~N} \mathrm{KCl}(\mathrm{pH}$ 7.4) in precooled teflon-coated glass homogenizer for few minutes to make $10 \% \mathrm{w} / \mathrm{v}$ tissue homogenate. Liver homogenate was centrifuged at $8000 \times \mathrm{g}$ for $15 \mathrm{~min}$ (at $4^{\circ} \mathrm{C}$ ) and the supernatant was subjected to ultracentrifugation (WX-Ultra 90, T-890 rotor, Thermoscientific, USA) at $78,000 \times \mathrm{g}$ for $90 \mathrm{~min}$. The supernatant thus obtained, was used as cytosolic fraction and a portion of it was undergone ultracentrifugation at $1,05,000 \times \mathrm{g}$ for $1 \mathrm{~h}$ to obtain microsomes (precipitate) to assay UDP-glucuronosyl transferase (UDPGT) activity, cytochrome P-450 (cyt P-450) content and to determine lipid peroxidation. The cytosolic fraction was used to determine glutathione Stransferase (GST) activity (Das et al., 2010).

\subsubsection{Assay for Cyt P-450, UDPGT and GST}

The total amount of microsomal cyt P-450 was estimated by the method as described (Zhang et al., 2012). The reaction mixture contained $1 \mathrm{~mL}$ sodium dithionite $(1$ $\mathrm{mg} \mathrm{mL}^{-1}$ ) and $1.8 \mathrm{~mL} \mathrm{CO}$-saturated water. The reaction was then started by addition of $0.2 \mathrm{~mL}$ microsomal enzyme preparation. The reaction mixture without the enzyme was used as blank. The absorbance was read at $450 \mathrm{~nm}$. The content of cyt P-450 was determined from the dithionite-reduced microsomes, with a molar extinction coefficient value of $91 \mathrm{~cm}^{-1} \mathrm{mM}^{-1}$.

UDPGT activity towards $p$-nitrophenol was determined according to the standard method (Zhang et al., 2013). The specific activity of UDPGT is expressed as activity/mg microsomal protein $/ \mathrm{min}$.

GST activity was measured (Olorunnisola et al., 2012) using 1-chloro-2, 4-dinitrobenzene (CDNB) as substrate. GST catalyzes formation of CDNB-GSH (reduced glutathione) conjugates to produce dinitrophenyl thioether, which is detected spectrophotometrically at $340 \mathrm{~nm}$. One unit of GST activity is defined as the amount of enzyme producing $1 \mathrm{mM}$ of CDNB-GSH conjugate/min under the conditions of the assay using molar extinction coefficient value of $9.6 \mathrm{mM}^{-1} \mathrm{~cm}^{-1}$.

\subsubsection{Lipid Peroxidation}

Lipid peroxidation in liver was estimated by the method available (Maia et al., 2010). Malondialdehyde (MDA), a product of lipid peroxidation was determined spectrophotometrically by using Thiobarbituric AcidReactive Substances (TBARS). Microsomal suspension $(0.2 \mathrm{~mL})$ of liver tissue sample was mixed thoroughly with $0.8 \mathrm{ml}$ of phosphate buffered saline $(\mathrm{pH} \mathrm{7.4)}$ and $0.025 \mathrm{~mL}$ of butylated hydroxytoluene solution $(8.8 \mathrm{~g}$ $\mathrm{L}^{-1}$ ). Then $0.5 \mathrm{~mL}$ of $30 \%$ trichloroacetic acid was added and sample was placed on ice for $2 \mathrm{~h}$ and centrifuged at $2000 \times \mathrm{g}$ for $15 \mathrm{~min} .1 \mathrm{~mL}$ of the supernatant was mixed with $0.25 \mathrm{~mL}$ of $1 \%$ thiobarbituric acid in $0.05 \mathrm{~N} \mathrm{NaOH}$ and $0.075 \mathrm{~mL}$ of $0.1 \mathrm{M}$ EDTA. After that, the samples were placed in boiling water for $20 \mathrm{~min}$ and allowed to cool to room temperature and the absorbance was determined at $532 \mathrm{~nm}$. Total TBARS were expressed as MDA, using molar extinction coefficient for MDA of $1.56 \times 105 \mathrm{~cm}^{-1} \mathrm{M}^{-1}$.

\subsubsection{Total Protein Determination}

Total protein was determined from cytosolic as well as microsomal fraction by modified Lowry protein assay kit.

\subsection{Statistical Analysis}

Statistical calculations were performed with graphpad Instat version 3.0 (graphpad software, Inc., San Diego, California, USA). Experimental data were statistically analyzed to find out differences between the data of the normal control rats and the other experimental groups of rats and between the experimental groups of rats, using Dunnett's t-test, following Analysis of Variance (ANOVA). Statistical level of significance was considered with a probability value $<0.05$.

\section{RESULTS}

\subsection{Average (Mean) Body Weights of Rats Belonging to Different Groups}

The average body weights of rats of different experimental groups on 0,7 th, 14th, 15th, 16th, 17th, 18th, 19th and 20th weeks are depicted in Fig. 1. At 20th week (4 weeks after STZ treatment), group II (diabetic control) and group III (carcinogen control) rats had significantly $(p<0.05)$ lower mean body weights than those of the rats of those two groups at the beginning of the experiment (i.e., week 0 ) and the mean body weight of normal control rats on the 20th week. 


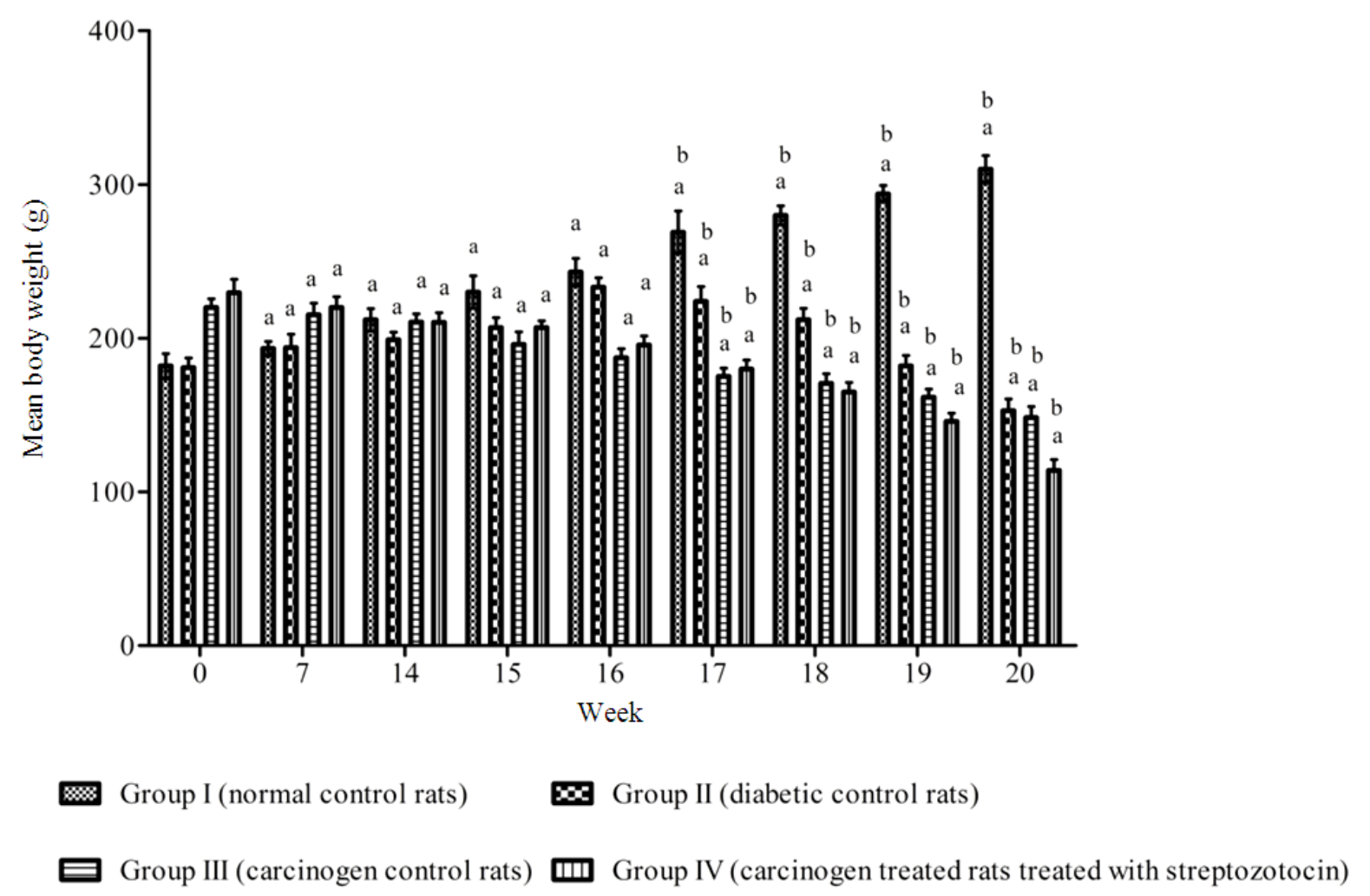

Fig. 1. Mean body weights of different experimental groups of rats. Each value represents mean $\pm \operatorname{SD}(n=6) .{ }^{a} \mathrm{P}<0.05,{ }^{b} \mathrm{P}<0.05$. ${ }^{\text {a }}$ Statistical level of significance (using Dunnett's $t$-test) when mean body weights of rats of different experimental groups on 7th, 14th, 15th, 16th, 17th, 18th, 19th and 20th weeks were compared with the data on 0 week. ${ }^{\text {b Statistical level of }}$ significance (using Dunnett's $t$-test) when mean body weights of rats on 17th, 18th, 19th and 20th weeks were compared with those at 16 th week

Interestingly, mean body weights in group IV rats (carcinogen treated rats treated with streptozotocin) were significantly $(\mathrm{p}<0.05)$ reduced as compared to group II (diabetic control) and group III (carcinogen control) rats. A remarkable reduction of weekly mean body weights has been observed in group IV rats (carcinogen treated rats treated with streptozotocin) from 17 th week (i.e., one week after the STZ administration).

\subsection{Changes in Cyt P-450 Content, some Enzyme Activities and Alteration in Lipid Peroxidation Level on the 20th week of the Experiment}

In group II (diabetic control) rats, hepatic cyt P-450 content was significantly $(\mathrm{p}<0.05)$ elevated. In group III (carcinogen control) rats, cyt P-450 content was significantly $(\mathrm{p}<0.05)$ decreased as compared to the normal control rats (group I). Cyt P-450 content in group
IV rats (carcinogen treated rats treated with STZ) was significantly $(p<0.05)$ elevated as compared to group III rats. However, the isoenzyme content of group III rats (carcinogen control) was lower than that of group II (diabetic control) rats (Fig. 2a).

The diabetic control (group II) and carcinogen control (group III) rats had shown an increase of hepatic UDPGT activity as compared to the normal control (group I) rats. Hepatic UDPGT activity was increased significantly $(\mathrm{p}<0.05)$ in carcinogen treated rats treated with STZ (group IV) as compared to carcinogen control (group II) rats (Fig. 2b).

GST activity in liver was markedly enhanced in diabetic control rats (group II) and carcinogen control rats (group III) as compared to group I rats. Interestingly, hepatic GST activity was significantly $(\mathrm{p}<0.05)$ elevated in carcinogen treated rats treated with STZ (group IV) as compared to group II and group III rats (Fig. 2c). 


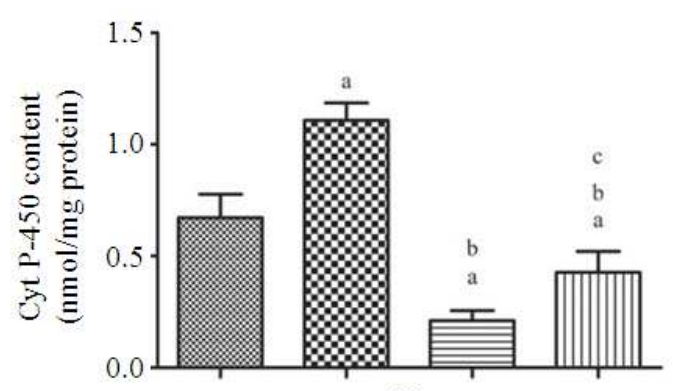

(a)

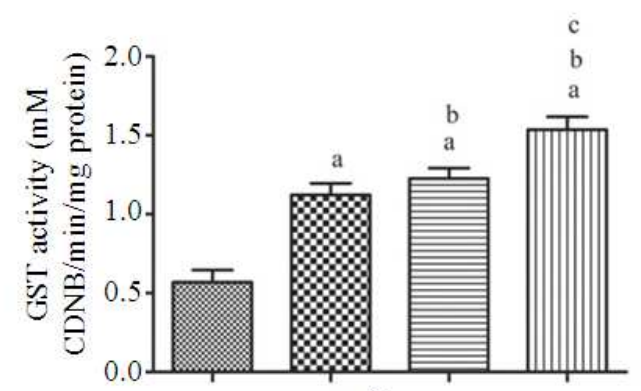

(c)

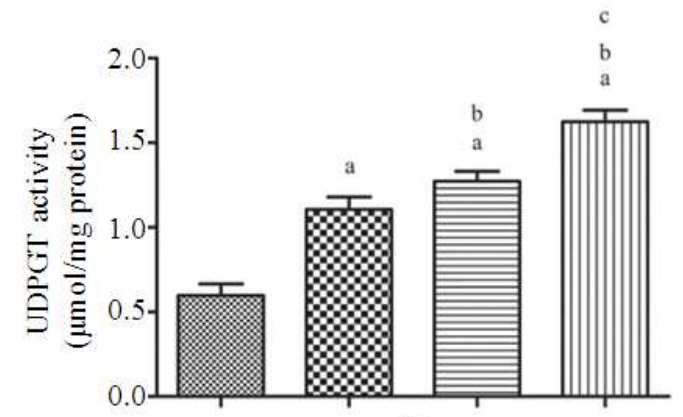

(b)

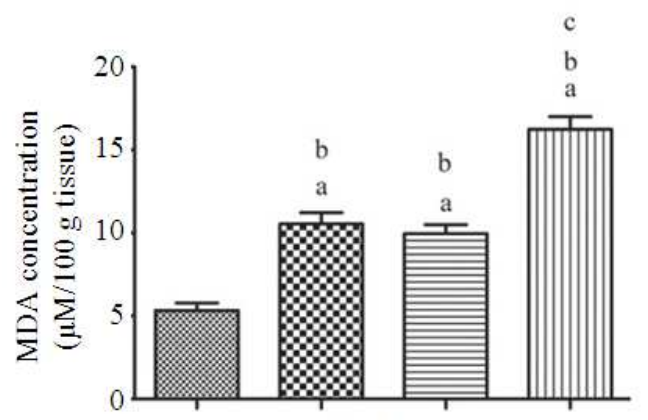

(d)

\footnotetext{
Group I (normal control rats)

$\mathbf{\infty}$ Group II (diabetic control rats)

曰Group III (carcinogen control rats) سI

Group IV (carcinogen treated rats treated with streptozotocin)
}

Fig. 2. Assessment of various biochemical investigations in livers of rats of different experimental groups. (a) Cyt P-450 content (b) UDPGT activity (c) GST activity (d) Lipid peroxidation level. Each value represents mean $\pm \mathrm{SD}(\mathrm{n}=6) .{ }^{\mathrm{a}} \mathrm{P}<0.05,{ }^{\mathrm{b}} \mathrm{P}<0.05$, ${ }^{\mathrm{c}} \mathrm{P}<0.05$. ${ }^{\mathrm{a}} \mathrm{Statistical}$ level of significance (using Dunnett's t-test) of the data obtained from rats belonging to groups II, III and IV, when compared with those data of group I rats. 'Statistical level of significance (using Dunnett's t-test) of the data obtained from group III and IV rats when compared against group II rats. 'Statistical level of significance (using Dunnett's t-test) of the data obtained from group IV rats when compared against group III rats

An elevation of MDA concentration was found in group II (diabetic control) and group III (carcinogen control) rats as compared to that of the normal control (group I) rats. However, a marked elevation $(\mathrm{p}<0.05)$ in lipid peroxidation (as assessed by MDA level) in the carcinogen treated rats treated with STZ (group IV) were observed as compared to carcinogen control (group III) rats (Fig. 2d).

\subsection{Histopathological Observations in Liver}

When normal control livers (livers of group I rats) were compared with STZ treated diabetic control livers (livers of group II rats), there was no major changes detected in hepatocytes except the appearance of few pycnotic nuclei and dilated sinusoidal spaces were visible in the hepatic tissues of diabetic control rats. Further, many of the nuclei in hepatocytes of diabetic control rats had taken more haematoxylin stain (Fig. 3a and b).

In carcinogen control liver (liver of group III rats), preneoplastic lesions were predominantly visible along with some specific clone of hepatocytes as a precursor of spongioblasts. There were small size ground glass lesions present throughout liver tissues (Fig. 3c and d). 


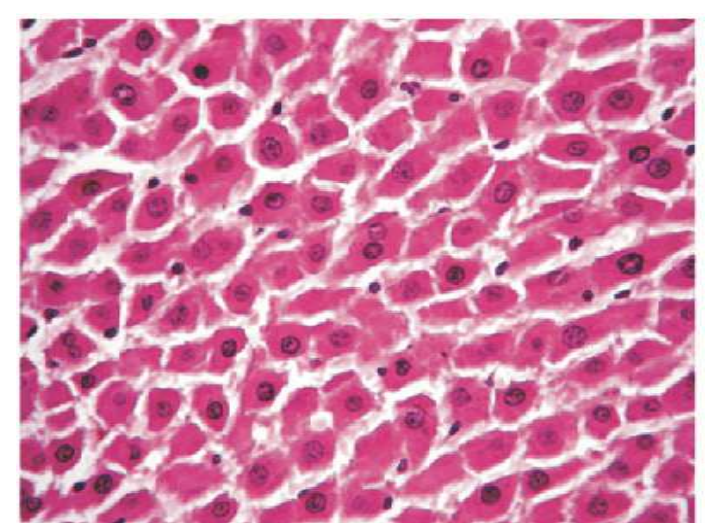

(a)

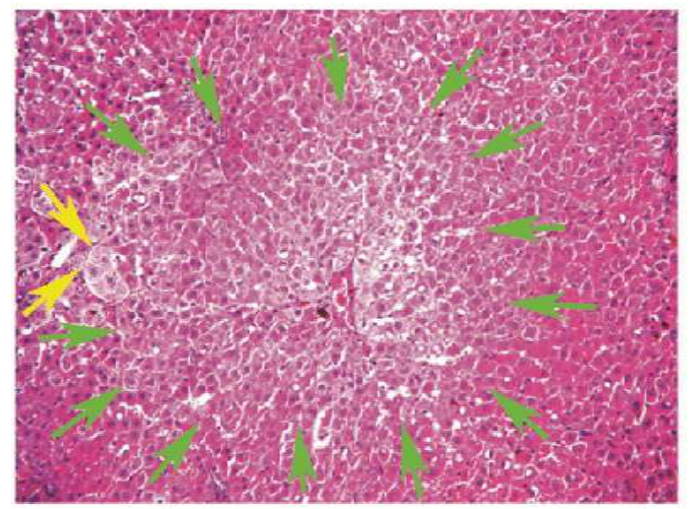

(c)

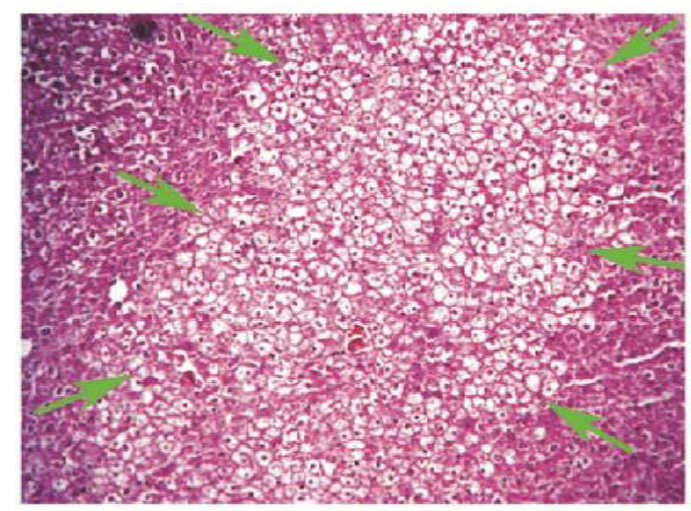

(e)

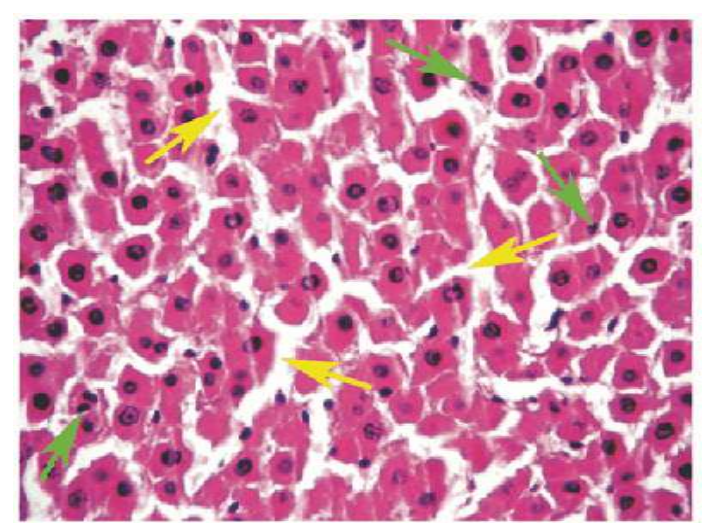

(b)

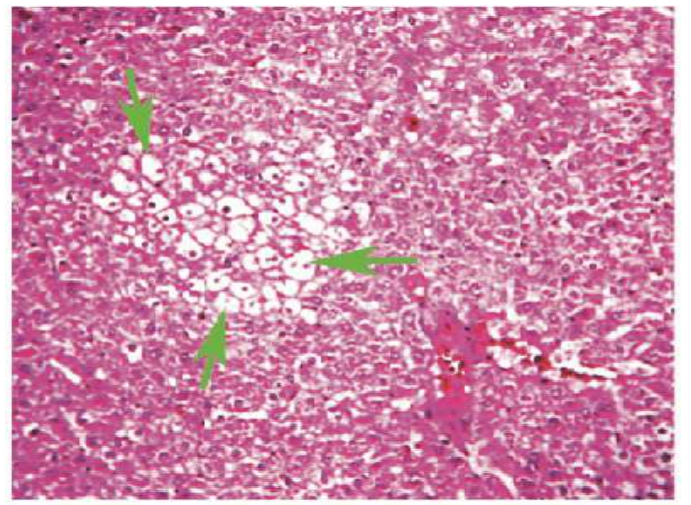

(d)

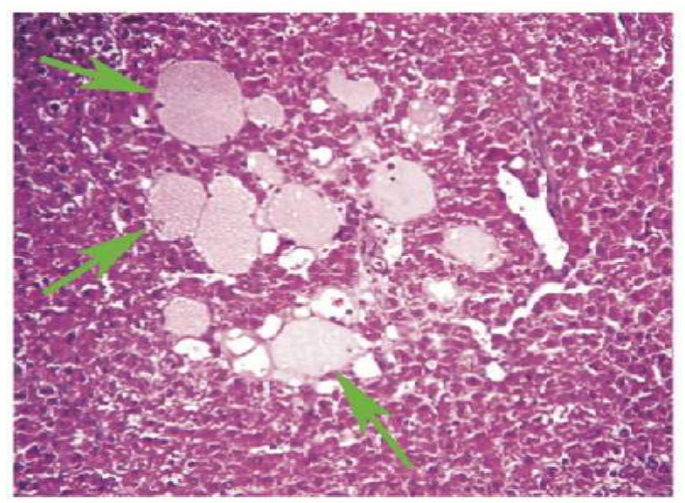

(f)

Fig. 3. Histological observations of different experimental rat livers ( $\mathrm{H}$ and $\mathrm{E}$ staining), (a) Liver section of a normal control rat showing normal hepatocytes (40×magnification), (b) Liver section of a diabetic control rat showing few hepatocytes containing pycnotic nuclei (shown by green arrows) and dilated sinusoidal space (shown by yellow arrows) in the hepatic tissue (40×magnification), (c) Liver section of a carcinogen treated rat showing development of large hepatic preneoplastic lesion (shown by green arrows) with the formation of specific clone of cells which generate spongioblasts (shown by yellow arrows) in hepatocytes (10×magnification), (d) Liver section of a carcinogen treated rat showing development of a preneoplastic ground glass lesion (shown by green arrows) in hepatocytes (10×magnification), (e) Liver section of a carcinogen treated rat treated with STZ, showing a large ground glass hepatic lesion (shown by green arrows) in hepatocytes (10×magnification), (f) Liver section of a carcinogen treated rat treated with STZ, showing formation of spongioblasts (shown by green arrows) in hepatocytes (10×magnification) 
In group IV rats (carcinogen treated rats made diabetic), there were many large ground glass lesions scattered in liver along with the formation of new small size hepatic lesions. The occurrence of formations of spongioblasts were also predominantly visible (Fig. 3e and f).

\section{DISCUSSION}

In this study, various biochemical alterations and histopathological changes have been demonstrated after induction of DM during the development of hepatocarcinogenesis in a defined rat model. Decreased body weight during DM generally occurs due to excessive breakdown of tissue proteins (Bonaldo and Sandri, 2013). Loss of body weight in cancer has also been known (Donohoe et al., 2011). In our study, a marked loss of body weights was observed in carcinogen treated rats following STZ administration as compared to the weight-loss observed in diabetic control rats and carcinogen control rats. This may be due to rapid progressive atrophy of adipose tissue and skeletal muscle after induction of DM during hepatocarcinogenesis. Cyt $\mathrm{P}-450$, an isoenzyme belonging to a supergene family of heme-containing oxidase, involved in the phase I drug biotransformation and plays a vital role in detoxification of many xenobiotics and endogeneous compounds in hepatocytes (Kulthong et al., 2012). The decrease in cyt P-450 content during hepatocarcinogenesis has been reported to alter the regulation of homeostasis, which affected both the expression of the monooxygenases and the growth controlling components of the preneoplastic cell population. STZ causes irreversible damages of the pancreatic $\beta$ cell causing DM (Yuan et al., 2010). The process of development of DM with STZ is much faster than that of chemically developed liver cancer. Obviously, chances of rate of generation of free radicals in DM are much more as compared to those in liver cancer. In carcinogen control animals, the cyt P450 content has been found to be even little less than $50 \%$ of the value of the isoenzyme as detected in the normal control rats. When carcinogen treated animals made diabetic with STZ, cyt P-450 content has become almost the double of that found in carcinogen control animals. The data suggests that in carcinogen control rats, the role of cytochrome enzymes might have been taken up by some other enzymes/enzyme system (s) or the carcinogen might have direct effect on reduction of cyt P-450 content. However, administration of STZ had an inductive effect on cyt P-450, which enhanced the level of cyt P-450 content more in STZ treated group IV rats. However, enhancement of cyt P-450 level was not sufficient enough to bring cyt P-450 level to its normal value. STZmediated elevation of cyt P-450 has already been in the literature (Chen et al., 2011). We confirm it again when cyt P-450 content data of diabetic control rats have been compared with those of the normal control rats.

UDPGT and GST, the two important phase II drug metabolizing enzymes, have important role in carcinogen metabolism and protection of liver against carcinogen insult (Chen et al., 2013; Zhang et al., 2013). They have been reported to increase their activities in preneoplastic and neoplastic lesions (Das et al., 2010). A report suggests that UDPGT activity was enhanced in liver of diabetic mice due to physicochemical changes in lipid composition and fluidity of microsomal membranes (Mukherjee et al., 2013). Enhancement of UDPGT activity and GST activity in diabetic control, carcinogen control and carcinogen treated rats made diabetic were shown similar trends. This suggests that the enzymes had predominant free radical scavenging roles both during the development of hepatocarcinogenesis and DM. Development of DM during hepatocarcinogenesis significantly enhanced these enzyme activities, suggesting that during the progression of DM in carcinogenic animals, generation of free radicals were much more and causing detrimental effect of DM on hepatocarcinogenic rats. The above explanation is further supported by the findings of lipid peroxidation level as measured by MDA concentration in hepatic tissue. MDA is a major end product of peroxidative degradation of the polyunsaturated fatty acid constituents of biological membranes and its mutagenic and carcinogenic properties have been shown in vitro and in vivo systems (Das et al., 2010). Diabetic control animals, carcinogen control animals and carcinogen treated animals made diabetic had $50 \%$ enhancement of lipid peroxidation levels than those found in diabetic control and carcinogen control animals, indicating the generation of much more free radical oxygen and lipid peroxides in those animals. The findings suggest that development of DM during progression of hepatocarcinogenesis definitely accelerate the process of development of liver cancer. Increased fatty acid oxidation in diabetic hepatocytes with an alteration of the enzymes involved in modulation of lipid peroxidation leads to 
proliferation of peroxisomes in hepatic parenchymal cells that even may be associated with the development of hepatocellular tumour (Nagarajan et al., 2012; Peyrou et al., 2012).

During comparison of hepatic histological architecture, no major difference was observed between normal and diabetic livers. However, few pycnotic nuclei along with more dilated sinusoidal space were predominantly visible in diabetic control hepatocytes. Nuclei took more basic stain suggesting that uncoiling of DNA and synthesis of mRNA were more. Darker stain in nucleus of the diabetic control hepatocytes indicate that the cells were engaged in growth and repairing activities (Settembre et al., 2012).

In carcinogen control rats, liver had number of preneoplastic and neoplastic lesions. Many of them had the ground glass appearance. Lesion areas were demarcated from the surrounding hepatocytes with the lack of eosin stain, indicating cytoplasms were more basophilic as compared to the cytoplasm of normal control and diabetic control hepatocytes. Further, spotty or less stained nuclei (vesicular type) were predominantly observed in the carcinogen control hepatocytes. Uncoiling of DNA disperses the stained molecules over a larger area and was not stacked up and the stained material (uncoiled DNA) was dispersed throughout the nuclear volume. DNA of those cells was uncoiled, suggesting cellular proliferation and excess protein synthesis as strongly basophilic nucleus is generally made of excess RNA. Basophilic cytoplasm as seen suggests unregulated cell division (Mills and Thomas, 2010). Thus the combination of basophilic cytoplasm and vesicular nucleus in carcinogen control hepatocytes reflects that the cells were not of normal type and were probably engaged in progress of the development of cancer in liver.

When carcinogen treated rats were made diabetic with STZ, the lesions were found to be much more in numbers (data not shown) and occupying larger area of liver. This suggests that DM enhances the progress of development of liver cancer. Further, in carcinogen control rats, there were only very few large hepatocytes observed. Those demarcated large hepatocytes were generally believed to produce spongioblast cells in liver which are considered to be the precursor of HCC. Carcinogen treated rats made diabetic (group IV rats) had many such spongioblast cells. This further strengthens that diabetic condition during the development of liver cancer expedites the process of development of the hepatocellular carcinoma.

\section{CONCLUSION}

The above mentioned observations in the defined animal model suggest that DM or persistent hyperglycemia during hepatocarcinogenesis promotes and accelerates progression of liver cancer. We believe, our study will provide a vivid understanding of these possible links between diabetes and $\mathrm{HCC}$, and this information may be relevant for the prevention and optimal patient management.

\section{ACKNOWLEDGEMENT}

Researchers are indebted to the grant of Department of Science and Technology (Government of India), Grant no. DST/Inspire Fellowship/2010/87.

\section{REFERENCES}

Bishayee, A., D. Bhatia, R.J. Thoppil, A.S. Darvesh, and E. Nevo et al., 2011. Pomengate mediated chemoprevention of experimental hepatocarcinogenesis involves Nrf2-regulated antioxidant mechanism. Carcinogenesis, 32: 888896. PMID: 21389260

Bonaldo, P. and M. Sandri, 2013. Cellular and molecular mechanisms of muscle atrophy. Dis. Model Mech., 6: 25-39. DOI: 10.1242/dmm.010389

Chen, G., P. Wang, G. Zhao, G. Xu and A. Gruzdev et al., 2011. Cytochrome P450 epoxygenase CYP2J2 attenuates nephropathy in streptozotocin-induced diabetic mice. Prostag. Oth. Lipid M., 2011 96: 6371. PMID: 21742052

Chen H.W., C.S. Huang, P.F. Liu, C.C. Li, C.T. Chen et al., 2013. Andrographis paniculata extract and andrographolide modulate the hepatic drug metabolism system and plasma tolbutamide concentrations in rats. Evid. Based Complement Alternat. Med., 2013: 1-11. PMID: 23997806

Das, T., F. Patra and B. Mukherjee, 2010. Effect of antisense oligomer in controlling c-raf.1 over expression during diethylnitrosamine-induced hepatocarcinogenesis in rat. Cancer Chemother Pharmacol., 65: 309-318. DOI: 10.1007/s00280009-1035-4 
Donohoe, C.L., A.M. Ryan and J.V. Reynolds, 2011. Cancer cachexia: Mechanisms and clinical implications. Gastroenterol. Res. Pract., 2011: 1-13. DOI: $10.1155 / 2011 / 601434$

Giovannucci, E., D.M. Harlan, M.C. Archer, R.M. Bergenstal and S.M. Gapstur et al., 2010. Diabetes and cancer: A consensus report. Diabetes Care, 33: 1674-1685. DOI: 10.2337/dc10-0666

Kulthong, K., R. Maniratanachote, Y. Kobayashi, T Fukami and T. Yokoi, 2012. Effects of silver nanoparticles on rat hepatic cytochr ome P450 enzyme activity. Xenobiotica, 42: 854862. PMID: 22458323

Liu, Y. and F. Wu, 2010. Global burden of aflatoxininduced hepatocellular carcinoma: A risk assessment. Environ Health Perspect., 118: 818-824. DOI: 10.1289/ehp.0901388

Liu, H., L. Liu, J. Li, D. Mei and R. Duan et al., 2012. Combined contributions of impaired hepatic CYP2C11 and intestinal breast cancer resistance protein activitiesand expression to increased oral glibenclamide exposure in rats with streptozotocininduced diabetes mellitus. Drug Metab. Dispos., 40: 1104-1112. PMID: 22393122

Maia, M.S., S.D. Bicudo, C.C. Sicherle, L. Rodello and I.C. Gallego, 2010. Lipid peroxidation and generation of hydrogen peroxide in frozen thawed ram semen cryopreserved in extenders with antioxidants. Anim Reprod. Sci., 122: 118-23. PMID: 20813469

Mills, O. and L.B. Thomas, 2010. Basaloid follicular hamartoma. Arch. Pathol. Laboratory Med., 134: 1215-1219. DOI: 10.1043/2008-0620-RS.1

Mukherjee, B., C.M. Hossain, L. Mondal, P. Paul and M.K. Ghosh, 2013. Obesity and insulin resistance: An abridged molecular correlation. Lipid Insights, 6: 1-11. DOI: 10.4137/LPI.S10805

Nagarajan, P., M.J. Mahesh Kumar, R. Venkatesan, S.S. Majundar and R.C. Juyal, 2012. Genetically modified mouse models for the study of nonalcoholic fatty liver disease. World J. Gastroenterol., 18:1141-1153. PMID: 22468076
Olorunnisola, O.S., G. Bradley and A. J. Afolayan, 2012. Protective effect of $T$. violacea rhizome extract against hypercholesterolemia-induced oxidative stress in wistar rats. Molecules, 17: 6033-6045. DOI: $10.3390 /$ molecules 17056033

Peyrou, M., P. Ramadori, L. Bourgoin and M. Foti, 2012. PPARs in liver diseases and cancer: Epigenetic regulation by microRNAs. PPAR Res., 2012: 1-16. PMID: 23024649

Settembre, C., R. Zoncu, D.L. Medina, F. Vetrini and S. Erdin et al., 2012. Lysosome-to nucleus signalling mechanism senses and regulates the lysosome via mTOR and TFE. EMBO J., 731: 1095-1098. DOI: 10.1038/emboj.2012.32

Simon, D. and B. Balkau, 2010. Diabetes mellitus, hyperglycaemia and cancer. Diab. Metab., 36: 182191. DOI: 10.1016/j.diabet.2010.04.001

Thomas, M.B., D. Jaffe, M.M. Choti, J. Belghiti and S. Curley et al., 2010. Hepatocellular carcinoma: Consensus recommendations of the national cancer institute clinical trials planning meeting. J. Clin. Oncol., 28: 3994-4005. DOI: 10.1200/JCO.2010.28.7805

Whiting, D.R., L. Guariguata, C. Weil and J. Shaw, 2011. IDF diabetes atlas: Global estimates of the prevalence of diabetes for 2011 and 2030. Diabetes Res. Clin. Pract., 94: 311-321. PMID: 22079683

Yuan, H.D. and S.H. Chung, 2010. Protective effects of fermented ginseng on streptozotocin-induced pancreatic $\beta$-cell damage through inhibition of NFKappa $\beta$. Int. J. Mol. Med., 25: 53-58. PMID: 19956901

Zhang, Y., Y. Li and Q. Li, 2012. Inhibition of cytochrome $\mathrm{P} 4503 \mathrm{~A}$ in rat liver by the diorganotin (IV) compound di-n-Butyl-di-(4 chlorobenzohydroxamato) tin (IV) and its probable mechanism. Molecules, 17: 10994-11009. DOI: 10.3390/molecules170910994

Zhang, C.L., T. Zeng, X.L. Zhao and K.Q. Xie, 2013. Garlic oil attenuated nitrosodiethylamine-induced hepatocarcinogenesis by modulating the metabolic activation and detoxification enzymes. Int. J. Biol. Sci., 9: 237-245. DOI: 10.7150/ijbs.5549 